\title{
Palliative surgery for giant mucinous carcinoma of the breast in an elderly patient: A rare case report
}

\author{
HARUKO TAKUWA, WAKAKO TSUJI and FUMIAKI YOTSUMOTO \\ Department of Breast Surgery, Shiga Medical Center for Adults, Moriyama, Shiga 524-8524, Japan
}

Received October 5, 2016; Accepted June 6, 2017

DOI: $10.3892 /$ mco.2017.1386

\begin{abstract}
Mucinous breast carcinoma (MBC) is relatively rare, accounting for $<10 \%$ of all breast cancers in women. These tumors are usually slow-growing and exhibit less aggressive characteristics compared with other types of breast cancer. Between 1989 and 2016, 55 patients underwent surgery for MBC at the Shiga Medical Center for Adults (Moriyama, Japan). The 10-year disease-free survival (DFS) and overall survival rates were 94.5 and $100.0 \%$, respectively. Specifically, the 10-year DFS rates of pure MBC (PMBC) and mixed MBC were 97.7 and $83.3 \%$, respectively. We herein report the case of a sizeable mucinous carcinoma causing rupture of the skin and bleeding due to tumor pressure. Palliative surgery was performed in order to remove the bleeding source after a total of 5 months of preoperative endocrine-based therapy. In conclusion, palliative surgery improved the patient's quality of life and may be a viable option for PMBC patients. The aim of the present study was to review the characteristics and management of these tumors, particularly in elderly patients.
\end{abstract}

\section{Introduction}

Mucinous breast carcinoma (MBC) of the breast is a special type of breast cancer that is characterized by the presence of carcinoma cells surrounded by large amounts of extracellular mucin (1). MBC may be encountered in all age groups, and the median age at presentation is 50-57 years (2-4). MBC comprises approximately $<10 \%$ of all invasive breast cancers. This type of tumor has an overall better prognosis and a

Correspondence to: Dr Haruko Takuwa, Department of Breast Surgery, Shiga Medical Center for Adults, 5-4-30 Moriyama, Moriyama, Shiga 524-8524, Japan

E-mail: st24057@yahoo.co.jp

Abbreviations: MBC, mucinous breast carcinoma; PMBC, pure type mucinous breast carcinoma; MMBC, mixed type mucinous breast carcinoma; IDC, invasive ductal carcinoma; OS, overall survival; DFS, disease-free survival

Key words: breast cancer, mucinous carcinoma, palliative surgery, elderly higher incidence among peri- and post-menopausal patients. Pathologically, there are two main subtypes of $\mathrm{MBC}$, namely pure type MBC (PMBC) and mixed type MBC (MMBC) (1). $\mathrm{PMBC}$ in particular is known to have a favorable prognosis compared with invasive ductal carcinoma (IDC) (4-9). We herein report a case of a giant $\mathrm{MBC}$ causing rupture of the skin and bleeding due to tumor pressure, and review the characteristics and palliative management of these tumors, particularly in elderly patients.

\section{Case report}

A 81-year-old Japanese woman presented to the outpatient clinic of the Department of Breast Surgery of the Shiga Medical Center for Adults (Moriyama, Japan) in January 2016 with a history of rapid enlargement of a right breast mass. The patient had noticed a mass 10 years prior. There was no family history of breast cancer. On physical examination, a large nodular mass was identified, measuring $18 \times 15 \mathrm{~cm}$, involving the entire right breast (Fig. 1A). There were also palpable right axillary lymph nodes, with the largest measuring $4 \times 3 \mathrm{~cm}$. The left breast was normal. A computed tomography (CT) scan revealed a large heterogeneous solid mass with axillary lymph node metastases (Fig. 1B); however, there was no evidence of distant metastasis.

PMBC with axillary lymph nodes metastases was diagnosed by core needle biopsy and fine-needle aspiration cytology. The patient received letrozole endocrine therapy as a primary systemic therapy, as she declined surgery. Soon after endocrine therapy initiation, the patient visited our emergency room due to continuous bleeding from lacerated skin caused by tumor pressure. The patient was found to be anemic (red blood cells $2.98 \times 10^{12} / \mu 1$, hemoglobin $8.4 \mathrm{~g} / \mathrm{dl}$ ), due to tumor neovascularization and intratumoral bleeding.

Laboratory data revealed marked elevation of the serum tumor markers carcinoembryonic antigen (CEA) and carbohydrate antigen $15-3$ (CA15-3) to $175.1 \mathrm{ng} / \mathrm{ml}$ and $196.0 \mathrm{U} / \mathrm{ml}$, respectively.

After 4 months, the tumor had increased in size, and the patient was started on exemestane and tegafur plus uracil (UFT ${ }^{\circledR}$; Taiho Pharma, Tokyo, Japan), as the tumor was considered difficult to control by endocrine therapy alone. The patient's advanced age was taken into consideration when selecting a chemotherapy regimen, in order to preserve her quality of life (QOL). A large clinical trial of UFT-based 
A

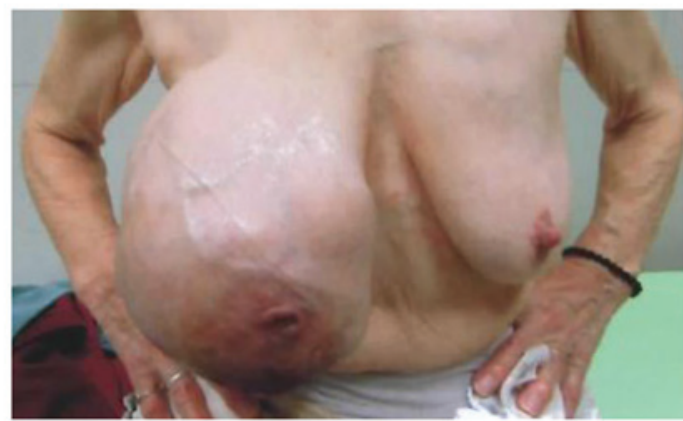

B

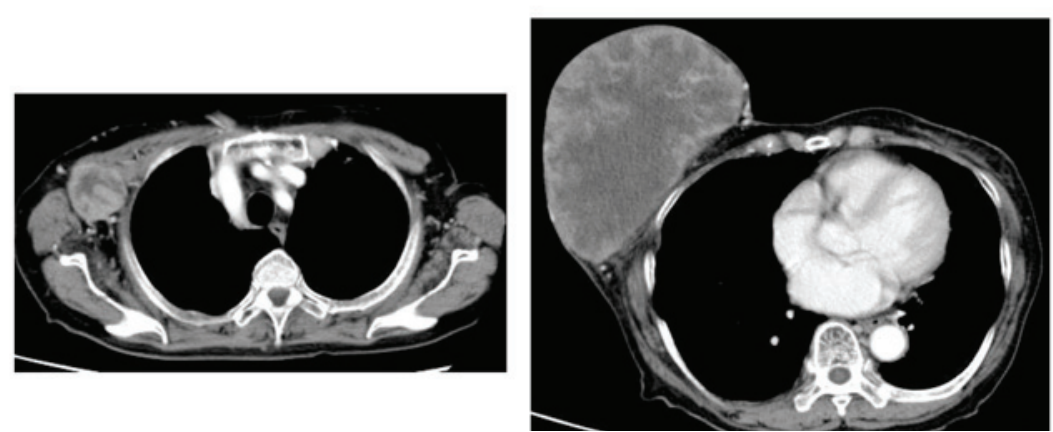

Figure 1. (A) On physical examination, the patient presented with a large nodular mass, measuring $18 \mathrm{x} 15 \mathrm{~cm}$, with severe disfigurement of the right breast. The contralateral breast was normal. (B) A computed tomography scan revealed a heterogeneous solid mass and lymph nodes metastases.

A
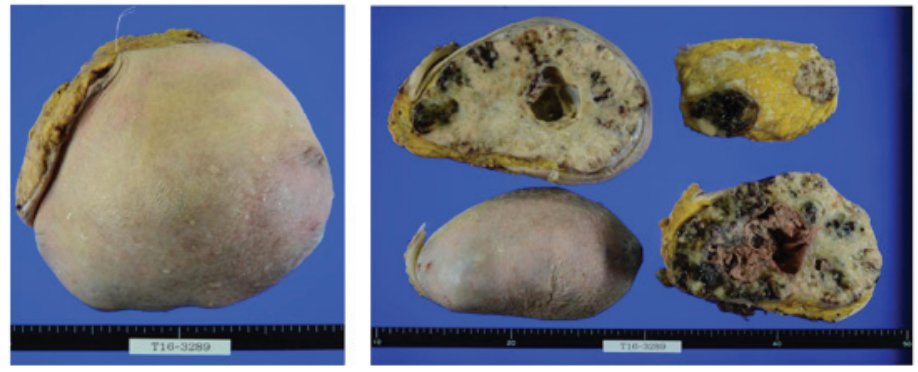

B
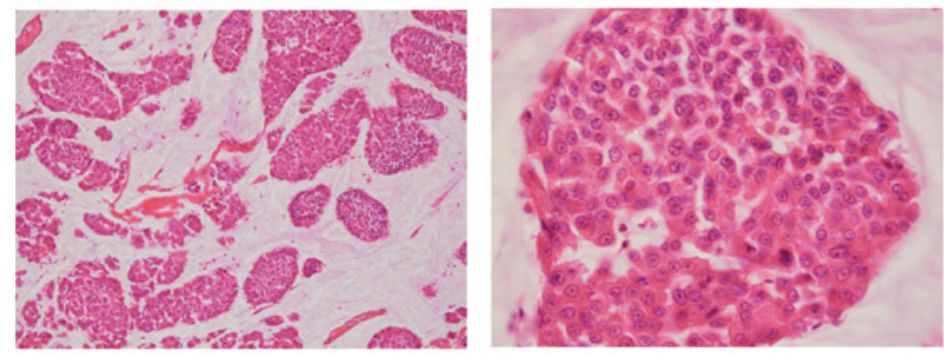

Figure 2. Pathological results of the postoperative specimen. (A) The excised mass was sized $18 \times 15 \mathrm{~cm}$. (B) Histological analysis of the surgical specimen. The diagnosis of pure type mucinous breast carcinoma was made using hematoxylin and eosin staining (left panel: Magnification, x100; right panel: Magnification, x400).

postoperative chemotherapy conducted in Japan (NSASBC-01 trial) demonstrated that UFT is useful for the treatment of intermediate-risk patients (10). At 4 weeks after the patient was started on second-line therapy, there was no significant reduction in the breast tumor. Finally, the patient consented to receive simple mastectomy. Despite the lymph node metastases, the axillary nodes were not removed, as this was a palliative surgery aiming to remove the bleeding source. The main purpose of the treatment for this patient was not to completely remove the macroscopically visible tumor, but maintain the patient's QOL and avoid breast cancer-related death. The surgically resected tumor was $1.96 \mathrm{~kg}$ in weight and $17.8 \mathrm{~cm}$ in diameter (Fig. 2A). The surgical margin of the breast was clear, but metastatic lymph nodes remained in the 
axilla. On histological analysis, the tumor was classified as a PMBC with mucinous differentiation in the histologically high-grade intraductal component, as determined by the accumulation of intraductal mucin (Fig. 2B).

The patient's anemia subsided after the bleeding source was removed (red blood cells $4.14 \times 10^{12} / \mu 1$, hemoglobin $12.5 \mathrm{~g} / \mathrm{dl}$ ). In addition, the tumor marker levels significantly decreased (CEA from 175.1 to $13.6 \mathrm{ng} / \mathrm{ml}$, and CA15-3 from 196.0 U/ml to $32.7 \mathrm{U} / \mathrm{ml}$ ) following mastectomy (Fig. 3). The patient remained well without evidence of distant metastasis after the surgery and, despite the metastatic axillary lymph nodes, lymphedema or neuropathy of the right arm has not appeared thus far (last follow-up, May 2017). The patient provided consent regarding the publication of the case details and associated images.

The medical records of $55 \mathrm{MBC}$ patients were retrospectively reviewed. Between 1989 and 2016, all the patients who underwent breast surgery at the Shiga Medical Center for Adults were investigated and $\chi^{2}$ tests were used to analyze qualitative data. Overall survival (OS) was defined as the period from the date of diagnosis to the date of the last follow-up or death from any cause, and disease-free survival (DFS) was defined as the period from the date of diagnosis to occurrence of any event, such as disease progression, relapse, recurrence or death. Kaplan-Meier estimates were used to calculate OS and DFS using Stat Mate V for Win \& Mac Hybrid software (ATMS, Tokyo, Japan).

\section{Discussion}

MBC represents 1-7\% of all breast cancers $(1,3,4)$ and is classified by the World Health Organization into two subtypes: i) PMBC if the non-mucinous component is $<10 \%$ and ii) MMBC if the non-mucinous component comprises $10-49 \%$ of the tumor. PMBC may be subtyped into a hypocellular variant (PMBC-A), exhibiting a tubular, cribriform, cord-like, micropapillary or papillary growth pattern, and a hypercellular variant (PMBC-B), growing in solid nests (1). It is generally accepted that PMBC has a favorable prognosis compared with IDC (2-9).

MMBC is mainly associated with lobular or ductal neoplasia (in situ or invasive), and a proportion of these tumors exhibit neuroendocrine differentiation. However, a specific percentage has not been clearly established for the diagnosis of MMBC. Due to the distinct clinicopathological characteristics of PMBC and MMBC, there may be a prognostic difference between the two types.

Locally advanced MBC is relatively rare. However, it was previously reported that there is no correlation between tumor size or subtype and prognosis in PMBC-A or PMBC-B. However, MMBC is known to have a prognosis similar to that of IDC. It has also been reported that breast-conserving surgery is effective for $\mathrm{MBC}$ due to their low local recurrence rate $(3,7,11)$.

In order to select the optimal therapy in rare cases, such as elderly patients with locally advanced MBC, our experience with this disease and the previous related literature was reviewed.

Between 1989 and 2016, 55 patients underwent breast surgery for mucinous carcinoma at the Shiga Medical Center for Adults. The mean patient age was 63 years (range, 37-85 years). Following surgery, the patients received thera-
TM

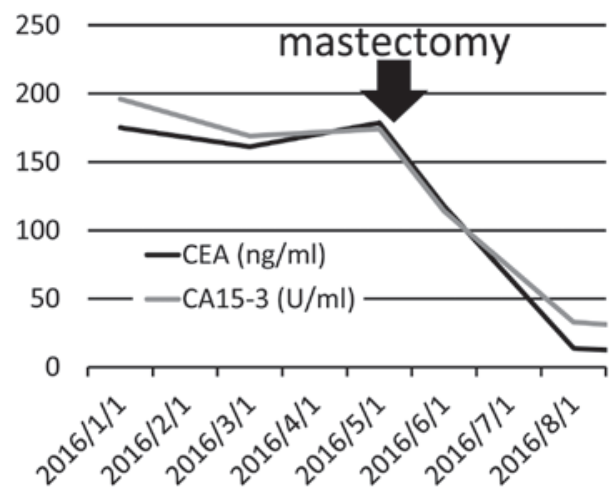

Figure 3. Tumor marker levels before and after treatment. TM, tumor marker; CEA, carcinoembryonic antigen; CA15-3, carbohydrate antigen 15-3.

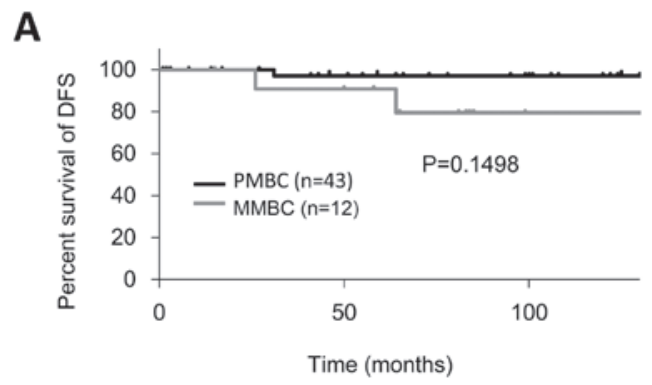

B

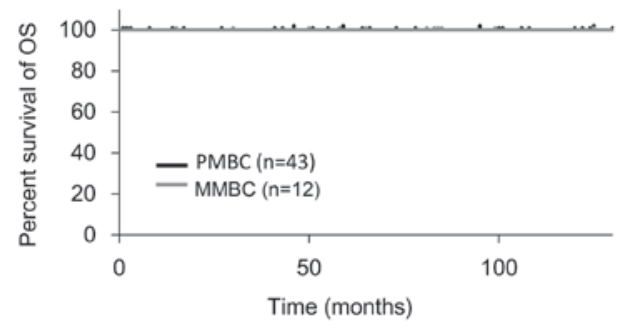

Figure 4. Kaplan-Meier estimates of (A) DFS and (B) OS of MBC patients. DFS, disease-free survival; OS, overall survival; MBC, mucinous breast carcinoma; $\mathrm{PMBC}$, pure type $\mathrm{MBC}$; MMBC, mixed type MBC.

pies administered according to the National Comprehensive Cancer Network guidelines, version 1.2015 (12).

Prior to 2,000, selective estrogen receptor modulator (SERM) therapy was selected for pre- and post-menopausal patients with estrogen receptor (ER)-or progesterone receptor $(\mathrm{PgR})$-positive cancer. Endocrine therapy was mainly administered for 2 years. However, in patients with high risk of recurrence, such as node-positive patients or those with tumors sized $>5 \mathrm{~cm}, 5$-year endocrine therapy with 2 years of UFT was used prior to 1995 , after which time 5-year hormonal therapy became the standard. Since 2000, aromatase inhibitors were used in post-menopausal hormonal receptor (HR) -positive patients for 5 years after surgery. Intravenous chemotherapy was administered in HR-negative patients and highly node-positive patients.

The 10-year DFS and OS were 94.5 and $100.0 \%$, respectively (Fig. 4). The 10-year DFS of PMBC and MMBC was 97.7 and $83.3 \%$, respectively. The clinicopathological characteristics are summarized in Table I. Statistically significant 
Table I. Clinicopathological variables of patients with mucinous breast carcinoma $(n=55)$.

MBC, no. (\%)

Variables

Age (years, mean $\pm \mathrm{SD}$ )

Tumor size $(\mathrm{mm}$, mean $\pm \mathrm{SD})$

Number of positive LN (mean \pm SD)

Sex

Female

Male

Axillary LN status

Negative

Positive

Unknown

TNM stage

I

II

III

ER status

Positive

Negative

Unknown

PgR status

Positive

Negative

Unknown

HER2 status

Positive

Negative

Unknown

Ki-67 expression, \%

$<20$

$\geq 20$

Unknown

Breast surgery

Mastectomy

Breast-conserving

Axillary surgery

Sentinel lymph node biopsy

Axillary lymph node dissection

No axillary surgery

Chemotherapy

No

Yes

Unknown

Radiotherapy

No

Yes

Unknown

Anti-HER2 target therapy

No

Yes
$\operatorname{PMBC}(n=43)$

$59.19 \pm 14.12$

$26.97 \pm 28.70$

$0.22 \pm 0.58$

42 (97.7)

$1(2.3)$

$36(83.7)$

$6(14.0)$

$1(2.3)$

19 (44.2)

$22(51.2)$

2 (4.6)

$27(62.8)$

6 (14.0)

$10(23.2)$

$22(51.2)$

$11(25.6)$

$10(23.2)$

1 (2.3)

18 (41.9)

24 (55.8)

$16(37.2)$

2 (4.7)

25 (58.1)

19 (44.2)

24 (55.8)

11 (25.6)

$28(65.1)$

4 (9.3)

$32(74.4)$

8 (18.6)

$3(7.0)$

18 (41.8)

$23(53.5)$

2 (4.7)

42 (97.7)

1 (2.3)
$\operatorname{MMBC}(\mathrm{n}=12)$

$59.25 \pm 15.24$

$32.00 \pm 19.64$

$1.25 \pm 3.14$

$12(100.0)$

$0(0.0)$

8 (66.7)

4 (33.3)

$0(0.0)$

4 (33.3)

$6(50.0)$

2 (16.7)

$9(75.0)$

$1(8.3)$

2 (16.7)

7 (58.3)

3 (25.0)

2 (16.7)

$1(8.3)$

7 (58.3)

4 (33.4)

5 (41.7)

$0(0.0)$

7 (58.3)

7 (58.3)

5 (41.7)

3 (25.0)

8 (66.7)

$1(8.3)$

7 (58.3)

4 (33.4)

$1(8.3)$

$6(50.0)$

2 (16.7)

4 (33.3)

$11(91.7)$

1 (8.3) 
Table I. Continued.

MBC, no. (\%)

Variables

$\operatorname{PMBC}(\mathrm{n}=43)$

$\operatorname{MMBC}(\mathrm{n}=12)$

Endocrine therapy

No

$11(25.6)$

2 (16.7)

Yes

$29(67.4)$

9 (75.0)

Unknown

Arm lymphedema

No

Yes

Unknown

MBC, mucinous breast carcinoma; PMBC, pure type MBC; MMBC, mixed type MBC; SD, standard deviation; LN, lymph node; TNM, tumor-node-metastasis; ER, estrogen receptor; PgR, progesterone receptor; HER2, human epidermal growth factor receptor 2.

differences were not observed between the characteristics of PMBC and MMBC. Of note, a male patient was included in the data. Although male $\mathrm{MBC}$ is rare, it has been previously reported (13-15).

Of the 55 patients, 3 developed recurrence. One patient had stage I, ER ${ }^{+} / \mathrm{PgR}^{+} / \mathrm{HER} 2-\mathrm{PMBC}$, and developed intramammary local recurrence 2 years and 7 months after breast-conserving surgery, followed by bone metastases at 8 years and 9 months after the operation. Second-line letrozole with zoledronic acid were continued, and the patient maintained stable disease at the last follow-up visit (May 2017). The remaining 2 patients experienced lung metastases: One patient had stage I ER ${ }^{+} / \mathrm{PgR}^{-} / \mathrm{HER} 2-\mathrm{MMBC}$, and developed multiple lung metastases 5 years and 4 months after the surgery. At the last follow-up visit (June 2017) the patient had progressive disease, controlled by 8th-line weekly paclitaxel. The second patient had stage IIIA (pt3n1M0) $\mathrm{ER}^{+} / \mathrm{PgR}^{+} / \mathrm{HER} 2^{-} \mathrm{MMBC}$, and developed lung and parasternal lymph node metastases 2 years and 2 months after the surgery. Surprisingly, at the last follow-up visit (May 2017) the patient had achieved clinically complete response by multidisciplinary therapy.

One of the PMBC patients suffered from arm lymphedema following axillary lymph node dissection. In the data presented herein, the incidence rate of patients who underwent axillary lymph node dissection was $2.8 \%$, which is relatively lower compared with previous reports $(5.3-54.0 \%)(14,16,17)$. The incidence rate of postoperative complications such as pain, lymphedema, numbness or motility disorders differs between sentinel node biopsy and axillary lymph node dissection $(16,17)$. It has been reported that chemotherapy with taxanes and radiation covering the breast and supraclavicular region were independent risk factors for lymphedema $(18,19)$. Generally, MBC is potentially resistant to chemotherapy or radiotherapy $(1,2,4)$. The combination of chemotherapy and radiation therapy with axillary dissection is infrequently selected, even in locally advanced $\mathrm{MBC}$, and we recommend that it is avoided.

Consistent with previous reports, the postoperative recurrence rate was higher in MMBC compared with PMBC (5,7-9).
Since distant metastasis is rare, particularly in PMBC, some reports observed no association between tumor size $(2,5,8)$ or lymph node status (8) and prognosis. It is considered that relapse develops after a long-term disease-free period, indicating that elderly PMBC patients do not always require aggressive chemotherapy or radical surgery. Even in patients with axillary lymph node metastasis, it may be considered a viable option to obtain sufficient symptom improvement by palliative rather than radical surgery.

If axillary lymph node status is not correlated with prognosis in PMBC, the main purpose of axillary surgery is staging and dissection may be omitted.

As in the present case, axillary lymph node dissection is not always considered necessary, as there was no complaint of arm edema or pain due to tumor pressure, and the patient continues systemic therapy after surgery. The patient's severe anemia improved immediately after the removal of the main tumor. Given the characteristics of PMBC, it is less likely to lead to immediate tumor-related death, and the possibility of arm edema due to direct vascular invasion from axillary lymph nodes is low.

In conclusion, palliative surgery may be a viable option, particularly for elderly patients with locally advanced PMBC, in order to maintain their QOL.

\section{References}

1. Rosen PP: Mucinous, Carcinoma Rosen's Breast Pathology. 3rd edition. Lippincott and Wiliams \& Wilkins, Philadelphia, pp515-pp177, 2009.

2. Bae SY, Choi MY, Cho DH, Lee JE, Nam SJ and Yang JH: Mucinous carcinoma of the breast in comparison with invasive ductal carcinoma: Clinicopathologic characteristics and prognosis. J Breast Cancer 14: 308-313, 2011.

3. Di Saverio S, Gutierrez J and Avisar E: A retrospective review with long term follow up of 11,400 cases of pure mucinous breast carcinoma. Breast Cancer Res Treat 111: 541-547, 2008.

4. Diab SG, Clark GM, Osborne CK, Libby A, Allred DC and Elledge RM: Tumor characteristics and clinical outcome of tubular and mucinous breast carcinomas. J Clin Oncol 17: $1442-1448,1999$.

5. Komenaka IK, El-Tamer MB, Troxel A, Hamele-Bena D, Joseph KA, Horowitz E, Ditkoff BA and Schnabel FR: Pure mucinous carcinoma of the breast. Am J Surg 187: 528-532, 2004. 
6. Lacroix-Triki M, Suarez PH, MacKay A, Lambros MB Natrajan R, Savage K, Geyer FC, Weigelt B, Ashworth A and Reis-Filho JS: Mucinous carcinoma of the breast is genomically distinct from invasive ductal carcinomas of no special type. J Pathol 222: 282-298, 2010.

7. Pan B, Yao R, Shi J, Xu QQ, Zhou YD, Mao F, Lin Y, Guan JH, Wang XJ, Zhang YN, et al: Prognosis of subtypes of the mucinous breast carcinoma in Chinese women: A population-based study of 32-year experience (1983-2014). Oncotarget 7: 38864-38875, 2016

8. Paramo JC, Wilson C, Velarde D, Giraldo J, Poppiti RJ and Mesko TW: Pure mucinous carcinoma of the breast: Is axillary staging necessary? Ann Surg Oncol 9: 161-164, 2002.

9. Zhang $M$, Teng XD, Guo XX, Zhao JS and Li ZG: Clinicopathological characteristics and prognosis of mucinous breast carcinoma. J Cancer Res Clin Oncol 140: 265-269, 2014.

10. Watanabe T, Sano M, Takashima S, Kitaya T, Tokuda Y, Yoshimoto M, Kohno N, Nakagami K, Iwata H, Shimozuma K, et al: Oral uracil and tegafur compared with classic cyclophosphamide, methotrexate, fluorouracil as postoperative chemotherapy in patients with node-negative, high-risk breast cancer: National Surgical Adjuvant Study for Breast Cancer 01 Trial. J Clin Oncol 27: 1368-1374, 2009.

11. Anan K, Mitsuyama S, Tamae K, Nishihara K, Iwashita T, Abe Y, Ihara T, Nakahara S, Katsumoto F and Toyoshima S: Pathological features of mucinous carcinoma of the breast are favourable for breast-conserving therapy. Eur J Surg Oncol 27: 459-463, 2001.

12. National Comprehensive Cancer Network Guidelines Version 1.2015 Panel Members. https://www.nccn.org. Accessed Aug 21, 2017.

13. Gupta K, Sharma S, Kudva R and Kumar S: Mixed mucinous and infiltrating carcinoma occurring in male breast- study of clinico-pathological features: A rare case report. J Clin Diagn Res 9: ED07-ED08, 2015

14. Pawar PS, Poflee SV, Pande NP and Shrikhande AV: Preoperative cytological diagnosis of mucinous carcinoma (MC) of male breast. J Cytol 33: 58-59, 2016.

15. Ishida M, Umeda T, Kawai Y, Mori T, Kubota Y, Abe H, Iwai M, Yoshida K, Kagotani A, Tani T and Okabe H: Mucinous carcinoma occurring in the male breast. Oncol Lett 7: 378-380, 2014.
16. Haid A, Kuehn T, Konstantiniuk P, Köberle-Wührer R, Knauer M, Kreienberg R and Zimmermann G: Shoulder-arm morbidity following axillary dissection and sentinel node only biopsy for breast cancer. Eur J Surg Oncol 28: 705-710, 2002.

17. Schrenk P, Rieger R, Shamiyeh A and Wayand W: Morbidity following sentinel lymph node biopsy versus axillary lymph node dissection for patients with breast carcinoma. Cancer 88: 608-614, 2000.

18. Kim M, Shin KH, Jung SY, Lee S, Kang HS, Lee ES, Chung SH, Kim YJ, Kim TH and Cho KH: Identification of prognostic risk factors for transient and persistent lymphedema after multimodal treatment for breast cancer. Cancer Res Treat 48: 1330-1337, 2016.

19. Swaroop MN, Ferguson CM, Horick NK, Skolny MN, Miller CL, Jammallo LS, Brunelle CL, O'Toole JA, Isakoff SJ, Specht MC and Taghian AG: Impact of adjuvant taxane-based chemotherapy on development of breast cancer-related lymphedema: Results from a large prospective cohort. Breast Cancer Res Treat 151: 393-403, 2015. 